\title{
Thin-sectioning of wet snow after flash-freezing
}

\author{
Jean-Bruno Brzoska, Cécile Coléou, Bernard Lesaffre \\ Météo-France, Centre National de Recherches Météorologiques, Centre d'Études de la Neige, \\ 1441 rue de la Piscine, 38406 Saint-Martin-d'Hères Cedex, France
}

\begin{abstract}
A new method for preparing thin sections of wet snow is described. Samples are flash-frozen to immobilise the liquid water content (LWC). Next, the pore space is filled with ester, and the entire sample is frozen to a solid, sliceable block. Micrographs of slices are obtained using transmitted and specular illumination. The proportion of LWC is measured on the micrographs and compared with LWC measured independently using calorimetry. Section analysis is both facilitated and complicated by small bubbles which form during sample preparation. Flash-freezing and bubble formation are discussed theoretically.
\end{abstract}

\section{INTRODUCTION}

Understanding and modelling percolation of water through snow requires knowledge of the liquid water distribution within the snow. However, simple sectioning of wet-snow samples is not possible, since wet snow contains a liquid phase. This paper presents a method of preparing wet-snow samples by flash-freezing, so that they can be sectioned and observed by standard techniques. In the paper, an explanation is given for choosing flash-freezing, followed by a description of its use, some results, and a discussion of the method's accuracy. A parallel is drawn with some recent work on the directional solidification of organic alloys.

\section{CHOICE OF METHOD}

Wet snow contains three phases of the same compound ice (ice, liquid water, vapour). Although thin-sectioning of dry (two-phase) snow has been taking place for over 50 years (Bader and others, 1939), sectioning of wet snow, which requires refreezing of the sample, is far more difficult: the different phases must remain distinguishable. We are not aware of any previous published work on the visualisation of three-phase slices (or sections) of wet snow.

One possible technique is to dye the liquid water prior to freezing and sectioning the sample. However, a growing crystalline phase generally expels soluble impurities such as dye towards the liquid side of the interface; these impurities concentrate ahead of the freezing front and may alter the dynamics of crystal growth by lowering locally the melting point of the liquid phase (Mullins and Sekerka, 1963). The use of extremely dilute fluorescent dyes might provide a solution. Some of them are detectable even at a monomolecular thickness (Akamatsu and Rondelez, 1991): the few molecules of dye which can "dissolve" into the growing ice front might be sufficient to allow discrimination of water from ice. However, fluorescence microscopy requires sophisticated and expensive equipment (especially a high-sensitivity video camera), all of which would need heavy modification to be operated properly in a cold laboratory at $-15^{\circ} \mathrm{C}$.
We have used another approach, the flash-freezing of the wet-snow sample, in order to give the artificially frozen water menisci a texture different from that of the naturally frozen grains and ice necks of the wet snow.

Under some conditions, the freezing of water can nucleate gas bubbles. The difference in texture arises from differences in the number and size of gas bubbles trapped in the ice. In general, increasing the freezing rate increases the number of bubbles and decreases their size (Brownscombe and Hallett, 1967; Geguzin and Deiuba, 1977). However, other parameters can also act on the formation of such bubbles; some of them will be discussed here.

Two-dimensional sections of these bubble zones can be observed using either thick sections (also called section planes) under specularly reflected light (metallographic technique) or thin sections (slices) under transmitted light. In principle, the observation of section planes is the more powerful technique since it allows, without further preparation, quick and serial image acquisition. However, the observation under reflected light of a section plane of ice presents serious experimental difficulties (see below). Consequently, most of the following work has used thin sections, a tedious but reliable technique.

\section{SOME EFFECTS OF FLASH-FREEZING}

The quick solidification of a melt is often reported to form a microcrystalline phase (Wilcox and Kuo, 1973). Such procedures are well known in biology (cryofixation) for freezing microscopic biological structures without damage before electronic microscopy observation (Bald, 1987), and in metallurgy (tempering). For instance, two-phase views of alloys were obtained after quenching (flash-freezing) by Mollard and Flemings (1967). They showed that a patchwork of microcrystals developed at the initial place where the liquid phase was present.

However, in the case of snow, a neighbouring solid phase (the snow grains) is always present. When compared to the "common" freezing nuclei (micron size) that are involved in heterogeneous nucleation, these grains can be considered as "infinite plane surfaces of ice", where crystal growth is ener- 
getically favoured. Moreover, heterogeneous ice nucleation takes time, typically minutes for millimetre drops of distilled water at $-20^{\circ} \mathrm{C}$ (Hobbs, 1974, p. 429). We will see later that the characteristic velocity for flash-freezing of a water meniscus from an existing snow grain is about $1 \mathrm{~m} \mathrm{~s}^{-1}$, which gives freezing times of the order of $1 \mathrm{~ms}$, negligible as compared to the heterogeneous nucleation time. When observing slices of flash-frozen wet snow under crossed polarisers, we did not see the characteristic "patchwork texture" of microcrystalline materials which is found in tempered alloys (Chalmers, 1964; Mollard and Flemings, 1967). The purity of atmospheric water is high compared to alloys (which generally contain desired impurities), and the expected density of large freezing nuclei is much lower for wet snow than for metallic alloys. This could explain the difference in texture observed between flash-frozen wet snow and tempered melted alloys; unlike snow, alloys contain many large nuclei on which microcrystals can form.

Experiments have recently been performed to study the formation of gas bubbles ahead of a freezing front, and their subsequent entrapment in the solid phase (Akamatsu and Faivre, 1996). Because of the low solubility of impurities in the solid, the dissolved gases concentrate ahead of a growing freezing front. If the freezing-front velocity is high enough, supersaturation of the gas can be reached and the nucleation of bubbles begins in the vicinity of the freezing front. Once a bubble is formed, it can absorb the excess of dissolved gas in the neighbouring liquid, whose melting point increases locally. This neighbouring liquid then freezes around the growing bubble. If the dynamics of both freezing and gas absorption match, a bubble will be entrapped inside the solid phase. Based on our observations, we believe this process takes place during flash-freezing of wet snow. Similar features of entrapped gas bubbles in the solid phase have been described for the ice-water system (Geguzin and Deiuba, 1977).

\section{EXPERIMENTS}

We conducted studies on artificial samples of homogeneous wet snow. They were prepared in a cold laboratory using the following procedure.

\subsection{Preparation and characterisation of wet-snow samples}

(1) Natural snow was sieved (mesh size $5 \mathrm{~mm}$ ) into a $25 \mathrm{~cm}$ cubic Plexiglas box with a perforated bottom. Sieving destroyed most previous bonding of the snow grains, creating a homogeneous sample.

(2) The box filled with sieved snow was put into an isothermal chamber held at $0^{\circ} \mathrm{C}$, described in Figure 1. After the sample was in thermal equilibrium, $0^{\circ} \mathrm{C}$ water was then filled in from the bottom until it overflowed the sample. This stage took around $10 \mathrm{~min}$.

(3) Snow grains were grown in the slush until the desired size was reached (Raymond and Tusima, 1979). Depending on the experiment, this took from $20 \mathrm{~min}$ to 3 days.

(4) Excess water was removed from the bottom of the box (elapsed time $\sim 10 \mathrm{~min}$ ), and the sample was allowed to evolve in the $0^{\circ} \mathrm{C}$ isothermal chamber for the desired time (from 1 hour to several days).

(5) The sample was then taken out of the $0^{\circ} \mathrm{C}$ chamber. It was placed in the cold laboratory (held during this stage between $-3^{\circ}$ and $-1^{\circ} \mathrm{C}$ ), and two horizontal cores were taken simultaneously at the same height from the snow sample. The former was used for a volume determination of liquid water content (LWC) by calorimetry (Boyne and Fisk, 1990); the latter was prepared for the thin-section experiments by flash-freezing. Moreover, a few grains were taken to compute the mean convex radius of curvature $\left\langle r_{\mathrm{c}}\right\rangle$ from their shadow (Brun and others, 1987).

\subsection{Flash-freezing procedure}

To ensure capturing the actual distribution of liquid water of the wet snow, the sample was flash-frozen in the sampler itself. Taking advantage of the high permeability of snow to fluids, the flash-freezing was achieved by pouring a cold liquid across the sample. We used for this purpose $n$-pentane, cooled at $-70^{\circ} \mathrm{C}$ by indirect contact with $\mathrm{CO}_{2}$ dry ice. This liquid presents the following advantages:

It is not miscible with water.

It remains liquid down to $-120^{\circ} \mathrm{C}$, and at temperatures near $0^{\circ} \mathrm{C}$ has a much higher vapour pressure than water. After freezing the sample, it can easily be removed by vacuum evaporation without the risk of etching the sample by sublimation.

Owing to its low viscosity, it can flow quickly (several $\mathrm{cm} \mathrm{s}^{-1}$ ) across the sample without inducing excessive

\section{cold room ambient air $\left(\mathrm{T}<0^{\circ} \mathrm{C}\right)$}

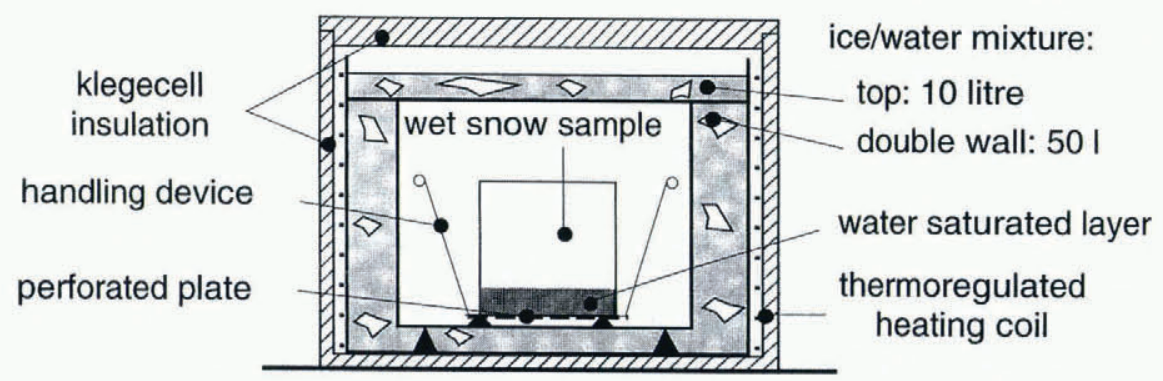

Fig. 1. Device to prepare wet snow in the cold laboratory. The heating coil controls the temperature of the air surrounding the ice/ water-filled container, which is kept at approximately $\pm 0.1^{\circ} \mathrm{C}$. This allows us to balance the natural thermal leakage of the $0^{\circ} \mathrm{C}$ chamber, regardless of the temperature of the cold room. 
shear stress on the snow-grains/liquid-water arrangements. At the observed filtration velocity $\left(\sim 5 \mathrm{~cm} \mathrm{~s}^{-1}\right)$, velocity in the pore space $(v)$ would be about $10 \mathrm{~cm} \mathrm{~s}^{-1}$. At this velocity, the characteristic dynamic pressure exerted by the pentane in the pore space $\left(\rho v^{2} / 2, \rho\right.$ being the density of $n$-pentane) is $5 \mathrm{~Pa}$, compared to the $\sim 600 \mathrm{~Pa}$ capillary pressure of a water meniscus of $0.25 \mathrm{~mm}$ mean radius.

It spreads naturally (Meunier, 1995) on both ice and water. The formation of "lenses" of coolant when the latter reaches water menisci is then avoided, which reduces the risk of deforming the menisci (Adamson, 1990).

\subsection{Thin-section making}

After flash-freezing, the pentane was removed by vacuum evaporation during $\sim 1 \mathrm{~min}$. The sample was then ready for sectioning. The experimental procedure used by the Swiss Federal Institute for Snow and Avalanche research at Davos (Good, 1987, 1989) was used. Briefly, since snow is a low-cohesion material, it has to be strengthened before cutting. This was achieved by filling all the open-pore volume with diethyl phthalate (DEP; purchased from Janssen Chimica, $\mathrm{C}_{6} \mathrm{H}_{4}\left(\mathrm{CO}_{2} \mathrm{C}_{2} \mathrm{H}_{5}\right)_{2}$, melting point $\left.-5^{\circ} \mathrm{C}\right)$ at a temperature between the melting temperatures of DEP and ice. The sample was then stored for a few hours at $-25^{\circ} \mathrm{C}$ to freeze the DEP, which is prone to supercooling.

A slice a few millimetres thick was sawn from a sample, stuck on a glass plate and surfaced with a sliding-blade microtome (Leica Histoslide 2000). This operation was repeated on the other side of the slice to obtain a thin section surfaced on both sides and attached to a flat glass substrate. To stick and unstick sections to the microtome, the DEP embedded in the sample was used as a glue. Sections were put on a Peltier thermoregulated plate. This plate was set to $-2{ }^{\circ} \mathrm{C}$ (above the melting point of phthalate) for unsticking, and $-15^{\circ} \mathrm{C}$ for sticking. A few drops of tetrahydronaphthalene (Tetralin; melting point $-35^{\circ} \mathrm{C}$ ) were added prior to covering the sample (with a glass plate) to dissolve and make transparent the filling DEP. The sample was then ready for observation.

To enhance the automatic detection of pores, the Tetralin was dyed with fat black for microscopy (Fluka, Nr 46300). It appeared dark blue once dissolved into the DEP.

\subsection{Image acquisition}

The observations were performed on a microscope Leica M420 using transmitted light. Images were taken using a Sony, $1024 \times 760$ pixels, 3CCD video camera, and were recorded on an analogue videodisc recorder. As explained in section l, a few images (details of the ice phase) have been taken at high magnification under specularly reflected light.

We had to observe scattered features, expected to cover only a few per cent of a total picture (ice + water zones + pores), and this picture had to be large enough to contain a statistically significant number of grains (about 100). Since the resolution of the camera is limited, a micrometric $x y$ displacement device was adapted to the macroscope. Composite pictures consisting of 16 separate images, each of $N_{\text {pix }}=768 \times 576$ pixels, were built. We have processed each of these 16 elementary pictures separately to analyze one thin section.

\section{RESULTS}

Twelve measurements were performed on six wet-snow samples prepared as described above, with LWC close to common values of irreducible water content and densities of $400-500 \mathrm{~kg} \mathrm{~m}^{-3}$. The last two samples (respective mean grain diameters 0.9 and $0.45 \mathrm{~mm}$ ) were sectioned at different depths. After a description of typical observations and density checks, the analysis of thin-section images is presented. Results are then compared to calorimetric LWC measurements. Quantitative data are summarised in Table 1.

Table 1. Fraction of liquid water "captured" by quick freezing

\begin{tabular}{|c|c|c|c|c|c|c|c|c|}
\hline \multirow{2}{*}{$\begin{array}{c}\text { Sample } \\
\text { No. }\end{array}$} & $\rho_{\mathrm{t}}$ & $\langle d\rangle$ & $e_{\mathrm{c}}$ & $\mathrm{LWC}_{\text {vol }}$ & $S_{\mathrm{b}} / N_{\text {pix }}$ & \multirow[t]{2}{*}{$e_{\mathrm{c}} /\langle d\rangle$} & \multirow{2}{*}{$\begin{array}{l}\Delta \\
\%\end{array}$} & \multirow{2}{*}{$\begin{array}{c}D_{\mathrm{c}} \\
\mathrm{mm}\end{array}$} \\
\hline & $\mathrm{kg} \mathrm{m}^{-3}$ & $\mathrm{~mm}$ & $\mu \mathrm{m}$ & $\%$ & $\%$ & & & \\
\hline 1 & 420 & $0.4^{*}$ & $70^{*}$ & 4.8 & 2.22 & 0.17 & 46 & n.d. \\
\hline 2 & 413 & 0.9 & $60^{*}$ & 3.0 & 1.88 & 0.07 & 63 & n.d. \\
\hline 3 & 415 & 0.5 & 20 & 4.3 & 2.26 & 0.04 & 53 & n.d. \\
\hline 4 & 526 & 0.8 & 30 & 3.9 & 1.08 & 0.04 & 28 & n.d. \\
\hline \multirow[t]{4}{*}{5} & 477 & 0.9 & 70 & 4.8 & 4.22 & 0.08 & 88 & 7 \\
\hline & 477 & 0.9 & 40 & 4.8 & 2.32 & 0.04 & 48 & 12 \\
\hline & 477 & 0.9 & 50 & 4.8 & 1.74 & 0.05 & 36 & 18 \\
\hline & 477 & 0.9 & 40 & 4.8 & 1.96 & 0.04 & 40 & 18 \\
\hline \multirow[t]{4}{*}{6} & 414 & 0.45 & 30 & 4.2 & 1.79 & 0.07 & 42 & 5 \\
\hline & 414 & 0.45 & 20 & 4.2 & 1.57 & 0.05 & 37 & 10 \\
\hline & 414 & 0.45 & $100^{*}$ & 4.2 & 2.52 & 0.23 & 60 & 20 \\
\hline & 414 & 0.45 & 50 & 4.2 & 1.04 & 0.11 & 24 & 25 \\
\hline
\end{tabular}

Notes: Temperature of flash-freezing: $-73<T<-67^{\circ} \mathrm{C} ; \rho_{\mathrm{t}}$ : total density of snow (including liquid water); $\langle d\rangle$ : mean grain diameter, defined by $\langle d\rangle=2\left\langle r_{\mathrm{c}}\right\rangle$, where $\left\langle r_{\mathrm{c}}\right\rangle$ is the average convex radius of curvature, computed from the shadow of $(3-\mathrm{D})$ isolated grains; $e_{\mathrm{c}}$ : section thickness; $\mathrm{LWC}_{\mathrm{vol}}$ : (volume of liquid water)/(total volume of sample); $S_{\mathrm{b}}$ : area of bubble zones (pixel) in the composite image; $N_{\text {pix }}$ : total area of the composite image; $\Delta=\left(1 / \mathrm{LWC}_{\mathrm{vol}}\right)\left(S_{\mathrm{b}} / N_{\text {pix }}\right)=$ (visualised water) $/$ (measured water); $D_{\text {c }}$ : cutting depth (from the top of the sample).

"Estimated value. n.d., no data.

\subsection{Example of thin-section observation}

Figure 2 presents different views taken from the same sample, a slice of flash-frozen snow $\sim 30 \mu \mathrm{m}$ thick. Figure $2 \mathrm{a}$ shows the composite view of the analyzed part $(7 \mathrm{~mm} \times$ $10 \mathrm{~mm}$ ) of the slice. The presence of several hundred snow grains ensures a statistically significant sample. Figure $2 \mathrm{~b}$ shows one of the 16 elementary images of Figure $2 \mathrm{a}$ at full resolution $(768 \times 576$ pixels $)$. The contouring of these bubble zones, which present an irregular texture, is not straightforward and will be detailed in the discussion. We have used colour separation to detect crudely but automatically (Fig. $2 \mathrm{~b}$ and $\mathrm{c}$ ) these regions, which appear grey under white transmitted light whatever the colour of the pore-space filler. Steep grain edges, which are dark and therefore present a low colour saturation, were removed by a two-pixel dilation (their contribution to water zones is negligible). A thresholding of the remaining grey tones gives (superimposed in black) an approximate visualisation of water zones. This procedure was used to validate the manual contouring used in this work (Fig. 2d).

Figure 3, taken from another part of the same slice, shows the appearance of the "grey zones" of the same pattern 


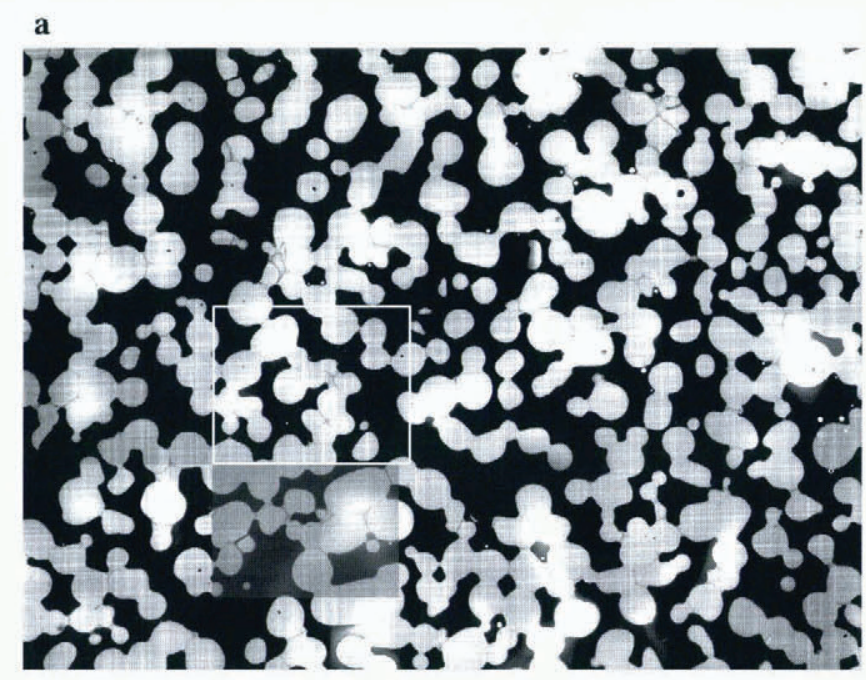

b
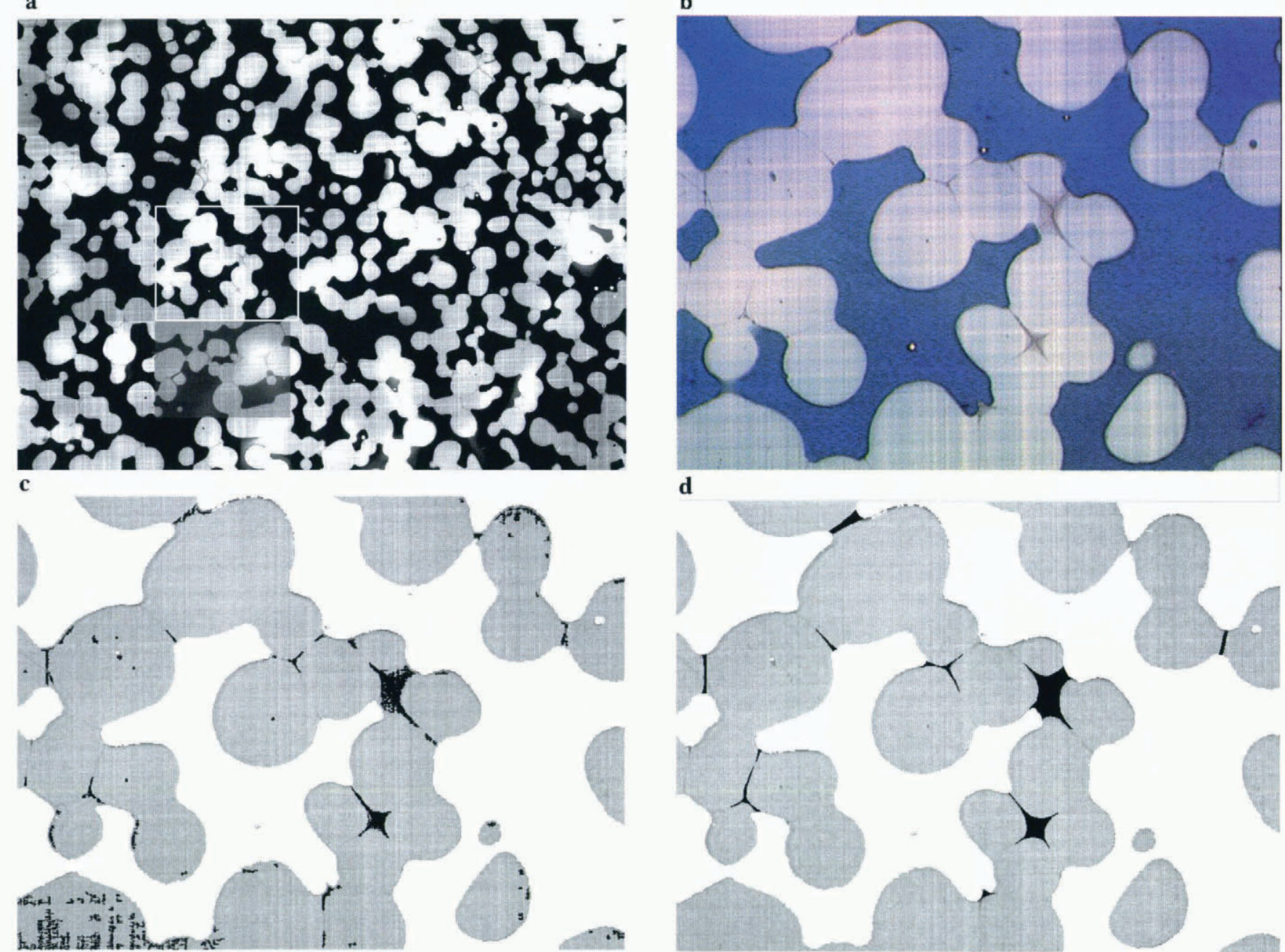

d

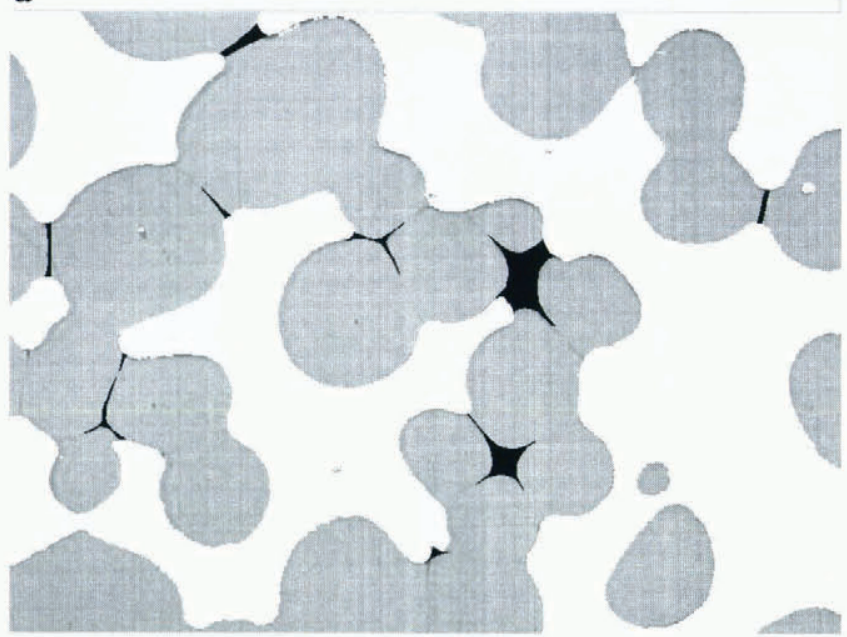

Fig. 2. Thin section of flash-frozen wet snow. Slice thickness $35 \mu \mathrm{m}$; mean grain diameter $0.8 \mathrm{~mm}$; flash-freezing temperature $-68^{\circ} \mathrm{C}$; views taken under non-polarised transmitted white light. (a) Composite view of 16 images, each $768 \times 576$ pixels in size. One of the images (white rectangle) is magnified in $(b)$. (b) Magnification of one image from $(a)$. The grains are white to grey; the water zones appear clearly as cross-like regions, plain grey or finely dotted. The dyed pore-filler appears blue. (c) Elimination of high-saturation regions. The visible "thread" of the picture is due to cutting defects (on both sides of the slice). (d) Manual contouring (superimposed in black).

under transmitted ordinary (Fig. 3a) and polarised (Fig. 3b) light. In these images, the grey or dotted patterns retain the same appearance: they darken the background. Therefore, these features are not made of microcrystals, but of bubbles. This interpretation is supported by the low colour saturation (i.e. a strong grey component) of these features. Concerning shapes, it can be noticed that:

The contours of bubble zones are curved and suggest capillary effects; they are consistent with the reported shape of water menisci in snow (Colbeck, 1979).

Conversely, the final features of grain boundaries are straight lines.

At triple points, tangents of grain and bubble-zone contours are different (see section 6.2).

Figure 4 shows a wet-snow sample (prepared in the same conditions as in Figure 2), slowly frozen in the cold laboratory at $-5^{\circ} \mathrm{C}$ instead of being flash-frozen: no bubbles can be seen.

\subsection{Density check}

Concerning isotropy, we did not observe anisotropy of either density or water menisci distribution, whatever the plane of sectioning for wet-snow samples (for dry snow, sieving introduced a vertical anistropy). Some test comparisons have been done between two-dimensional (pixel count) and three-dimensional (weighing) density of wet snow. Colour separation of slice images gave the area $S_{\mathrm{p}}$ (in pixels) of the pore space (Fig. 2d; region depicted in white). It was the region of high colour saturation (blue in our experiments). The two-dimensional density $d(2-\mathrm{D})$ was given by $d(2-\mathrm{D})=d_{\mathrm{i}}\left[1-\left(S_{\mathrm{p}} / N_{\text {pix }}\right)\right]$, with ice density $d_{\mathrm{i}}=0.917$. For these tests, the difference between $d(2-\mathrm{D})$ and $d(3-\mathrm{D})$ was of the order of $\pm 5 \%$, within the estimation of digitisation errors of our images.

\subsection{Comparison with bulk LWC measurements}

From the determination of the contours of bubble zones, the number of pixels $S_{\mathrm{b}}$ inside these contours was computed, giving the estimated area of the slice occupied by bubbles.

The ratio $S_{\mathrm{b}} / N_{\text {pix }}$ gives the estimated two-dimensional density of liquid water for the considered thin section. Assuming the isotropy of our samples, the density remains invariant from 2-D to 3-D (Serra, 1982, p. 253-254), and $S_{\mathrm{b}} / N_{\text {pix }}$ can be compared to the volume LWC of the sample, whatever the shape of water menisci. The only requirement is that the number of objects should be statisti- 
a

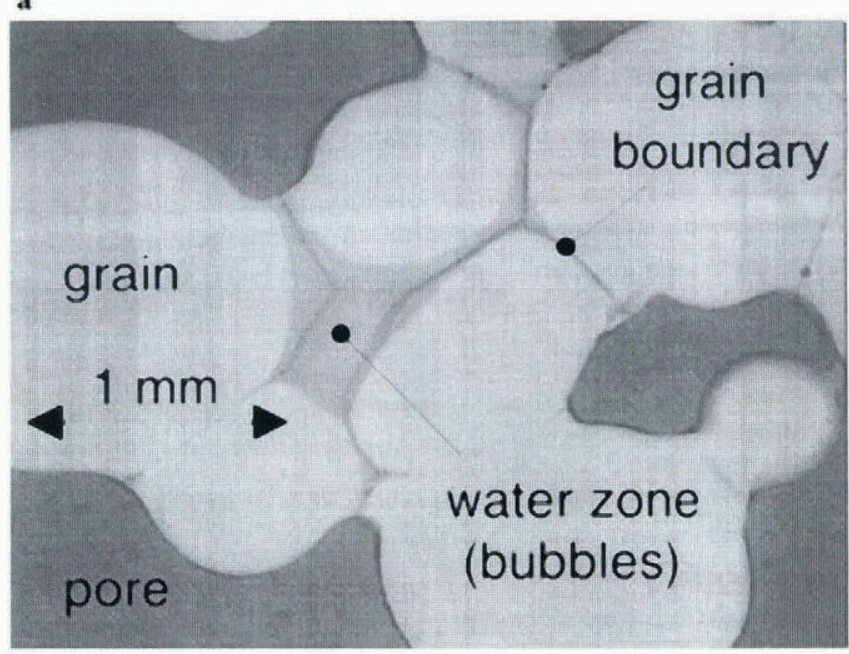

b

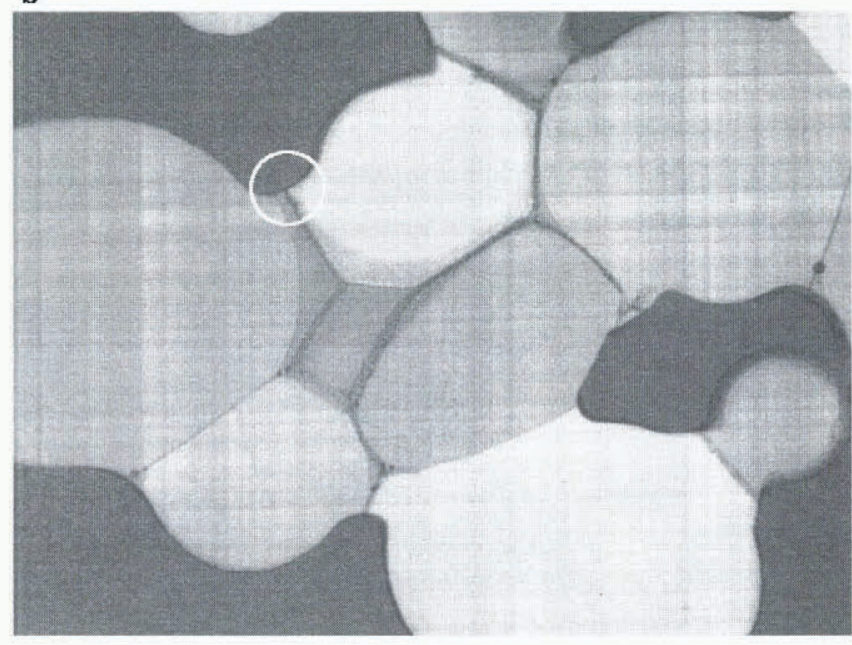

-Fig 3. Detail view of another part of the slice used for Figure 2, at a higher magnification, under different kinds of illumination. (a) Transmitted ordinary light. The variability of the texture of water zones (plain grey or finely dotted), which complicates image processing, appears clearly. However, the contours of these zones remain sharp at a local scale (a few pixels); this allows accurate manual contouring. (b) The same view under polarised light; the bubble patterns are kept unchanged by changing the orientation of the polariser. It can be noticed (white circle) that the contours of these patterns are not tangent to grain contours at the grain/bubble/pore junction.

cally sufficient. This was the case for our composite pictures which always contained more than 200 grains. Comparison of $S_{\mathrm{b}} / N_{\text {pix }}$ to calorimetric LWC measurements was done for four samples. For each of them, the flash-freezing was done using pentane at $-67^{\circ}$ to $-73^{\circ} \mathrm{C}$, and the thin sections were cut near (generally less than $1 \mathrm{~cm}$ from) the side of the sample which was first reached by the pentane. To clarify the influence of pentane temperature, thin sections have been made at different depths $D_{\mathrm{c}}$, starting from the top of samples (which had first received cold pentane). The results are given in Table 1. For the few points for which the cutting depth $D_{\text {c }}$ is known, $S_{\mathrm{b}} / N_{\text {pix }}$ decreases when increasing $D_{\mathrm{c}}$ (except for sample 6 at $D_{\mathrm{c}}=20 \mathrm{~mm}$, coming from a thick slice; see section 5.2). In all cases, $S_{\mathrm{b}} / N_{\text {pix }}$ was lower than bulk LWC. The mean observed difference between these two measurements was greater than the reported absolute error of the freezing calorimetry technique of LWC measurement (Boyne and Fisk, 1990).

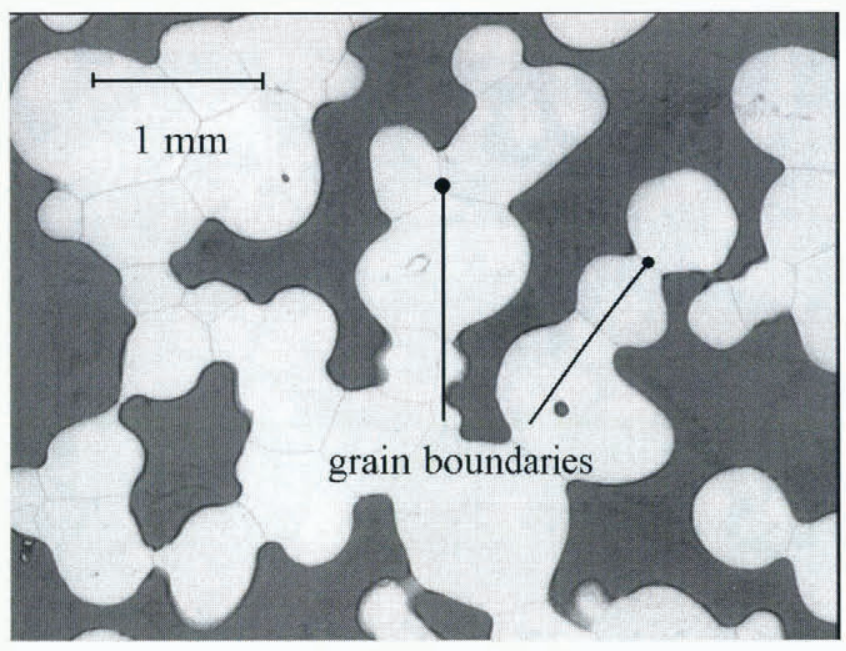

Fig. 4. A similar sample (thickness $\sim 30 \mu \mathrm{m}$ ), previously allowed to freeze slowly in the cold laboratory $\left(1 \mathrm{~h}\right.$ at $\left.-5^{\circ} \mathrm{C}\right)$. No dotted region can be seen.

\section{EXPERIMENTAL DISGUSSION}

The flash-freezing of wet snow produces zones of bubbles between grains, and these zones clearly suggest the shape of original water menisci. However, comparison of the area extent of bubbles with LWC measurements shows that the bubble areas do not define the entire liquid water area. These findings suggest that side effects of flash-freezing a material which initially contains a liquid phase cannot be neglected a priori. A discussion of potential errors resulting from these side effects follows.

\subsection{Errors related to image processing}

\section{(a) Digitisation errors}

Since the determination of bubble zones required some manual contouring, the impact of digitisation errors is difficult to define. However, it seems reasonable to assume that where sharp contrasts existed (at least at the scale of a few pixels, which is the case of our bubble-zone contours; see Fig. $2 b)$, the accuracy of manual contouring was \pm 1 pixel. If this was the case, then examining the number of pixels belonging to the bubble zone (see Fig. 2d) through a onestep erosion or one-step dilation image processing provides an estimation of digitisation effects. These effects are relatively large for thin zones (few pixels are concerned) and moderate for massive zones. On ten images (each of $768 \times 576$ pixels) from the same composite view, the numbers of bubble-zone pixels of respectively one-step eroded $\left(S_{\mathrm{e}}\right)$, normal $\left(S_{\mathrm{n}}\right)$ and one-step dilated $\left(S_{\mathrm{d}}\right)$ were compared. The ratios $\left(S_{\mathrm{n}}-S_{\mathrm{e}}\right) / S_{\mathrm{n}}$ and $\left(S_{\mathrm{d}}-S_{\mathrm{n}}\right) / S_{\mathrm{n}}$ remained of the order of 0.3. Assuming a drawing accuracy within 1 pixel (what is gained or lost by one-step erosion or dilation), one obtains an estimated digitisation error of $\pm 15 \%$.

\section{(b) Automatic vs manual detection of bubble zones}

Colour decomposition was used to select the "greyest" pixels, more exactly the lowest colour-saturation pixels. Since saturation is a measurement of the colour level (in our colour system, RGB, mostly the level of the "blue" canal), this discrimination is not accurate for dark (all values close to 0 ) or pale (R, G, B close to 255) patterns: the number of available colour levels is too small (Sève, 1996). 
The contours of grains, always dark even at high values of illumination (close to the video saturation of the CCD camera), were generally included in a set of "greyest" pixels. They were partly removed by eroding this set (see Fig. 2c). However, because of the variability of greytones in bubble zones (some of them were dark), the extensive discrimination of bubble zones was not possible using colour decomposition.

We were not able to apply the standard methods of texture analysis, such as covariance (Serra, 1982, p. 280-282), radial distribution function (Coster and Chermant, 1989, p.245) or histogram of random line intercepts (Eicken, 1993), since bubble zones could appear as either plain grey (bubble texture finer than the image resolution) or finely dotted (bubble texture can be seen) regions. For instance, these two textures are present in Figure 3a.

Finally, a manual contouring of bubble zones has been used in this work (Fig. 2d); the automatic detection using colour decomposition (incomplete; see Fig. 2c) was used as a validation. Manual discrimination of bubble zones was possible because the boundary between these zones and the rest of the grains was sharp. Whatever the texture and local grey tone of a zone, when compared to the neighbouring grains, the boundary remained sharp within the "mesh" of the bubble-zone texture. Because of the occurrence of dotted features, the classical edge-detection algorithms (gradient, etc.) could not be used, and because of the need to detect a sharp boundary, we could not use any preliminary smoothing procedure. As a further improvement, a fully automatic detection algorithm would have to combine texture analysis (isotropic) and edge detection (one of the possible textures being "plain grey"). Because of the large-scale variability of the background grey tones (illumination, dyeing conditions), this algorithm might scan the image with small subsets (typically $50 \times 50$ pixels), working separately on each of them.

\subsection{Possible errors coming from slice thickness}

From a single sample (grains of $0.5 \mathrm{~mm}$ diameter), we have prepared two sections of different thickness (20 and $100 \mu \mathrm{m})$; the views were recorded at the same magnification. We did not measure significant difference in grain area (two-dimensional density). Even if the bottom and top section planes are different, internal reflection allows a full illumination of the upper surface. However, the use of such "thick" sections may be misleading, because bubbles can be entrapped in grain boundaries without quick freezing (Hobbs, 1974, p. 328). If a grain boundary has a tilted position in the section, such bubbles (typical diameter $10 \mu \mathrm{m}$ ) could be confused with bubbles due to flash-freezing (Fig. 5). For a thin enough section ( $\leq 1 / 10$ of grain radius), these "natural" bubbles generally appear as a line of "large" bubbles at grain boundaries, and cannot be confused with flashfreezing bubbles. For the samples listed in Table 1, the ratio $e_{\mathrm{c}} /\langle d\rangle\left(e_{\mathrm{c}}\right.$ and $\langle d\rangle$ being respectively section thickness and mean grain diameter) was in most cases less than 0.1. Therefore, these sections were thin enough not to introduce additional errors into $S_{\mathrm{b}}$ as a result of grain-boundary bubbles or superposition of flash-freezing bubbles at different depths. On the contrary, the point of Table 1 for which $e_{\mathrm{c}} /\langle d\rangle=$ 0.23 (sample 6) clearly overestimates the area of bubble zones.

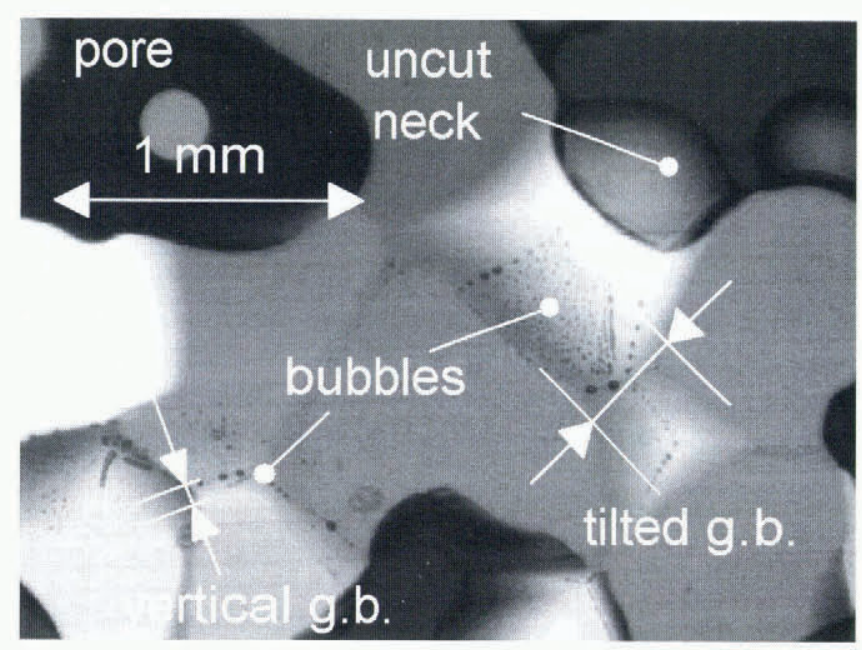

Fig. 5. Thick section $(150 \mu \mathrm{m})$ under polarised transmitted light: mean grain diameter $0.7 \mathrm{~mm}$; sample slowly frozen at $-5^{\circ} \mathrm{C}$. The bubbles are much bigger than those produced by quick freezing, and are always located inside regions of colour gradation. These regions, denoted between arrows on the picture, are vertical or tilted grain boundaries (g.b.).

\subsection{Sample deformation during flash-freezing}

Flash-freezing is a strong thermal shock. Because of the expansion of water, it is possible that grain deformation can occur during flash-freezing. If it does, such deformation would most probably occur during the freezing of the water between grains and would lead to cracks, because at this rapid rate of freezing $(\ll 1 \mathrm{~s})$, ice can be considered a brittle material. Such cracks were not observed in the ice phase (grains or menisci) of our thin sections. Also, for similar samples, we observed little difference in grain structure between flash-frozen and slowly frozen samples (cf. Figs $2 b$ and 4). Moreover, we did not notice changes in grain shape between two sections made in the same sample, one near the top and the other near the bottom. It seems that the risk of meniscus displacement by freezing is minimal.

\section{BUBBLE FORMATION PROCESS}

\subsection{Efficiency of freezing}

The formation of bubbles in the freezing water menisci is governed by the local conditions of solidification, i.e. the velocity of the freezing front. This velocity depends upon the temperature profile of the water in contact with pentane, and this profile depends upon the instantaneous temperature profile in the flowing pentane.

\section{(a) Warming of pentane}

As pentane flows across the snow sample (initially at $0^{\circ} \mathrm{C}$ ), it gets warmer. This problem is similar to the calculation for heat exchangers in laminar flow (because of the size of pores in snow, flow is laminar). Consider a pore network of parallel tubes of constant diameter $d$ (a classic industrial exchanger). For such problems, heat engineering provides the notion of thermal mixing length $\ell_{\mathrm{m}}$ (Eckert and Drake, 1972), defined as:

$$
\ell_{\mathrm{m}}=\frac{\lambda U d^{2}}{D_{\mathrm{th}}}
$$

where $\lambda$ is a numerical coefficient ( $\sim 0.05$ for a cylinder $), U$ is 
the mean fluid velocity in the tube (here $\sim 0.1 \mathrm{~m} \mathrm{~s}^{-1}$ ), and $D_{\text {th }}$ is the fluid thermal diffusivity $\left(\sim 10^{-7} \mathrm{~m}^{2} \mathrm{~s}^{-1}\right.$ for pentane).

Practically, one can consider that below a pentane path length of $\ell_{\mathrm{m}}$ (along a pore), there remains in the centre of the flow some liquid at its initial temperature $T_{0}$. Within this length, the presence of a heat sink at the known temperature $T_{0}$ can be assumed, and the estimate of the freezing velocity of water remains possible. In our case, one obtains for $\ell_{\mathrm{m}}$ a value of $\approx 1 \mathrm{~cm}$. According to our few results at different depths (samples 5 and 6), it appears that most of the sections have been made beyond the effective thermal mixing length of our system.

\section{(b) Velocity of freezing}

Since quick freezing is needed to form bubbles, we look for a conservative estimate of the freezing-front velocity. The slowest rate of freezing is expected when the cold pentane has to supercool the whole meniscus depth $e$ before the freezing begins.

We have not heard of an analytical solution to this problem. However, there exist experimental laws giving the freezing velocity $v$ vs the supercooling $\Delta T$, and the mean bubble diameter $d_{\mathrm{b}}$ vs $v$ (Hobbs, 1974, p. 584, 620-621).

Because of the no-slip condition of hydrodynamics, $v$ is very low near the meniscus (moreover, $e \ll d$ ). We can assume that this zone is governed by diffusion (this situation leads to the slowest freezing rate).

The freezing begins once the supercooling reaches the grain (this statement is supported by experiments). At this moment, the water layer presents an "S curve" temperature profile similar to the steady profile ahead of a freezing front growing in a supercooled liquid.

Finally, the thermal diffusivities of liquid water and alkanes are close together. One can therefore assume that the steady temperature profile of supercooled water does not change at the approach of the liquid-water/pentane interface.

Consequently, it seems reasonable to assume that the velocity of freezing in the menisci $v$ is close to the free growth velocity described by Hobbs. A direct extrapolation of the curves gives $v \sim 1 \mathrm{~m} \mathrm{~s}^{-1}$ for $\Delta T=50^{\circ} \mathrm{C}$ with a mean bubble diameter of $d_{\mathrm{b}} \sim 0.7 \mu \mathrm{m}$ (just large enough to be seen in visible light).

\subsection{Delay in bubble formation}

The process of bubble formation and entrapment described in section 2 (Akamatsu and Faivre, 1996) could explain the discrepancy between the area of bubble zones and LWC. The nucleation of bubbles needs a local supersaturation of gas in the liquid phase; the occurrence of such a supersaturation is preceded by a transient growth stage, which is necessary to accumulate enough gas ahead of the freezing front. During this stage, bubbles do not materialise, and so it becomes an invisible zone. By increasing the rate of the freezing process, one can shrink this "invisible" freezing zone (Hobbs, 1974, p. 601).

These explanations are supported by the few measurements made at different depths of a flash-frozen sample. Far from the top of the sample, the pentane, warmed up by its flow across the upper part of the sample, applies a weaker thermal gradient to liquid parts. The lower induced peak of dissolved gas concentration ahead of the freezing front explains a longer delay in bubble formation.

The geometry of bubble zones also supports the above explanations of "missing water". At $T=T_{\mathrm{d}}=0^{\circ} \mathrm{C}$, the receding contact angle of water on ice is zero, and on the contact line, ice/water, ice/pore and water/pore interfaces should present the same tangent plane. On a two-dimensional section plane, these interfaces should therefore present the same tangent. In general, this was not the case in our experiments (see Fig. 3b). This suggests that something has delayed the formation of bubbles. However, this argument would be restricted to capillary water menisci, and could not account for the thin film of water that is presumably present around the convex parts of grains. This water also contributes to LWC, but probably no more than $10-15 \%$. For large grains and at a low cutting depth (sample 5 in Table 1) the proportion $\Delta$ of visualised LWC reached $88 \%$.

Naturally, all these arguments assume a freezing front moving from existing grains towards the water/pore interface of menisci. This is consistent with thermodynamics (existing snow grains are low-curvature ice surfaces) and supported both by observations under polarised light (Fig. $3 \mathrm{~b}$; no change of crystal orientation from plain grain to bubble zone) and by "streetlike" alignments of bubbles (Fig. 6) parallel to the direction of freezing (Carte, 1961).

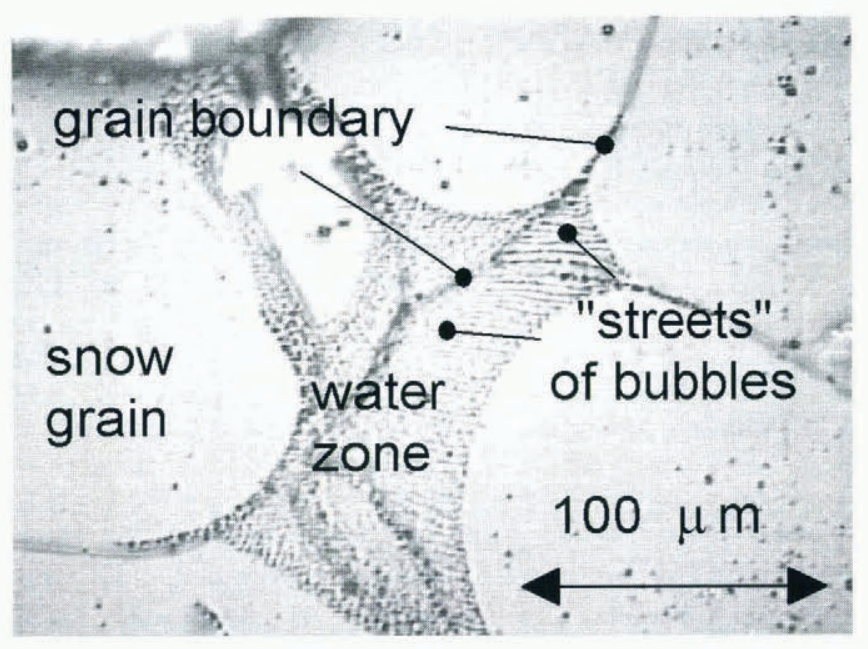

Fig. 6. Wet-snow sample, flash-frozen at $-70^{\circ} \mathrm{C}$, viewed in specularly reflected light (metallographic microscope, magnification $\times 200$ ), which shows the nature of these dotted regions.

\subsection{Serial cuts}

Besides having experimental difficulties, the above technique of thin-sectioning destroys at least the neighbouring $5 \mathrm{~mm}$ in the sample. Therefore, it is impossible to obtain serial cuts of a sample by this method. To obtain the experimental three-dimensional files of water menisci necessary to understand and model water percolation at fine scale, a means of "scanning" massive samples of flash-frozen wet snow must be designed.

Serial cuts allowing observation of bubbles within the ice phase require specularly reflected light. Under such conditions of illumination, freshly cut samples generally present a haze of small dots all over ice surfaces, which impedes 
the acquisition of clear bubble images. In the range of temperature $T$ of the cold laboratory $\left(-12^{\circ}\right.$ to $\left.-30^{\circ} \mathrm{C}\right)$ and microtome blade velocity $V\left(\sim 1\right.$ to $\left.50 \mathrm{~cm} \mathrm{~s}^{-1}\right)$ used in our experiments, the density of dots decreased with both $V$ and $T$ (the necessity of a low-speed cutting had already been noticed by Good (1987)), but not enough to make the images clear. These dots, present immediately behind the blade whatever the ambient humidity of the cold room, seem to be caused by gas bubbles rather than rime. We suspect the mechanism of their formation is bubble nucleation in liquid water (see Fig. 7) generated by the friction of the cutting blade (Colbeck, 1992).

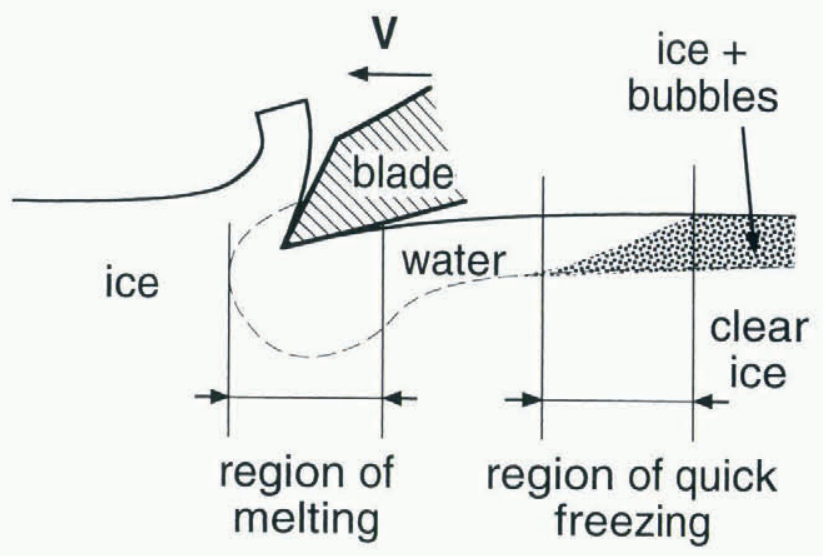

\begin{abstract}
Fig. 7. A possible explanation of the bubble formation during cutting. Both compression (which lowers the melting point) and blade friction (which increases the temperature) act commonly to form a liquid film close to the bulk ice, able to capture ambient air: the subsequent quick freezing of the film would produce the surface bubbles. It is difficult to measure the thickness of the altered region. When the experiment was performed at $-15^{\circ} \mathrm{C}$ (cold laboratory dew point $\sim-35^{\circ} \mathrm{C}$ ), we observed a sublimation rate of $\sim 1 \mu \mathrm{m} \mathrm{min}^{-1}$ and the dotted layer disappeared in $\sim 15 \mathrm{~min}$. At $-15^{\circ} \mathrm{C}$, the layer of surface bubbles was probably $10-20 \mu \mathrm{m}$ thick.
\end{abstract}

Sublimation in the cold laboratory $\left(\sim 10 \mathrm{~min}\right.$ at $-15^{\circ} \mathrm{C}$; ambient dew point $T_{\mathrm{d}} \sim-35^{\circ} \mathrm{C}$ ) is sufficient to remove these dots, but creates an uneven ice surface from which it is impossible to take images in specularly reflected light. We could obtain a few images of the only ice phase (at high magnification such as Figure 6). They gave valuable information on flash-freezing processes, but did not allow the systematic analysis of a whole section plane of snow, which contains entangled phases of ice and DEP. If we could use the same (low) magnifications as used for thin sections, but still use reflected light, serial cuts (three-dimensional) could also be obtained. Cutting at lower temperature $\left(\mathrm{T} \ll-20^{\circ} \mathrm{C}\right.$ ) may provide an answer to the "dot" problem. Experiments are now in progress in this direction.

\section{CONGLUSION}

The main conclusion from the above experimental discussion is the persistence of a discrepancy between LWC deduced from thin sections and calorimetric measurements. Flash-freezing is not a suitable method for LWC measurements. Our practical motivation in this work was to locate and describe accurately the most probable paths of percolation water in already wet snow. At least for low percolation rates (the most frequent case in the field), these channels are expected to connect some of the existing water menisci that have the largest section. We have shown that flash-freezing is able to visualise these wide menisci, i.e. the interesting data for percolation modelling. However, the "resolution" (in terms of menisci section) needs to be estimated.

More than potential errors related to digitisation, a physical reason is suspected: the existence of a transient stage between the beginning of the freezing and the nucleation of first bubbles. Some measurements performed at different depths of flash-frozen samples support this statement: the "visualisation efficiency" of flash-freezing decreases with the applied thermal gradient (in deeper regions of the sample).

Concerning visualisation techniques, the feasibility of observation under specularly reflected light has been shown at high magnifications, and the problem of surface defects of freshly cut ice has been addressed. We believe that a solution to this problem would provide a much more practical method for extensive study of wet snow than the difficult and time-consuming technique of thin sections. Moreover, unlike thin sections, the observation of a massive section of flash-frozen wet snow might allow in principle the three-dimensional reconstruction of water menisci. In the more distant future, "remote" tomographic techniques such as nuclear resonance microscopy (able to distinguish ice from water) might perform such a reconstruction. However, a very careful (and difficult) control of equilibrium conditions of wet snow $\left(T=T_{\mathrm{d}}=0^{\circ} \mathrm{C}\right)$ would be necessary throughout the time of measurement.

\section{ACKNOWLEDGEMENTS}

We thank W. Good, G. Krüsi, P. Duval, S. Akamatsu, J. Meunier and R. Blanchard for fruitful discussions.

\section{REFERENCES}

Adamson, A. W. 1990. Physical chemistry of surfaces. Fifth edition. New York, etc., John Wiley and Sons.

Akamatsu, S. and G. Faivre. 1996. Residual-impurity effects in directional solidification: long-lasting recoil of the front and nucleation-growth of gas bubbles. 7. Phys. I (Paris), 6, 503-527.

Akamatsu, S. and F. Rondelez. 1991. Fluorescence microscopy evidence for two different LE-LC phase transitions in Langmuir monolayers of fatty acids. f. Phys. II (Paris), 1, 1309-1322.

Bader, H., R. Haefeli, E. Bucher, J. Neher, O. Eckel and C. Thams. 1939. Der Schnee und seine Metamorphose. Beitr. Geol. Schweiz, Ser. Geotech. Hydrol. 3. (SIPRE Transl. 14, 1954.)

Bald, W. B. 1987. Quantitative cryofixation. Bristol, Adam Hilger.

Boyne, H. S. and D. J. Fisk. 1990. A laboratory comparison of field techniques for measurement of the liquid water fraction of snow. CRREL Spec. Rep. 90-3.

Brownscombe, J. L. and J. Hallet. 1967. Experimental and field studies of precipitation particles formed by the freezing of supercooled water. Q.7. R. Meteorol. Soc, 93 (398), 455-473.

Brun, E., F. Touvier and G. Brunot. 1987. Experimental study on thermal convection and grains picture analysis. In Jones, H. G. and W. J. Orville-Thomas, eds. Seasonal snowcovers: physics, chemistry, hydrology. Dordrecht, etc., D. Reidel Publishing Co., 75-94. (NATO ASI Series C: Mathematical and Physical Sciences 211.)

Carte, A. E. 1961. Air bubbles in ice. Proc. Phys. Soc. (London), 77 (495), 757-768.

Chalmers, B. 1964. Principles of solidification. Secondedition. New York, etc., Wiley and Sons.

Colbeck, S. C. 1979. Grain clusters in wet snow. 7. Colloid Interface Sci., 72 (3), $371-384$.

Colbeck, S. C. 1992. A review of the processes that control snow friction. 
CRREL Monogr. 92-2.

Coster, M. andJ. L. Chermant. 1989. Précis d'analyse d'image. Paris, Presses du CNRS.

Eckert, E. R. G. and R. M. Drake. 1972. Analysis of heat and mass transfer. Tokyo, McGraw-Hill.

Eicken, H. 1993. Automated image analysis of ice thin sections - instrumentation, methods and extraction of stereological and textural parameters. J. Glaciol., 39 (132), 341-352.

Geguzin, Ye. E. and A.S. Deyuba. 1977. Videleniye gaza, formirovaniye i zakhvat gazovyhk puzirkov na fronte kristalizatsii iz razplava [Gas emission, formation and catching of gas bubbles from the liquid phase into a front of crystallisation]. Kristallografiya, 22 (2), 348-353.

Good, W. 1987. Thin sections, serial cuts and 3-D analysis of snow. International Association of Hydrological Sciences Publication 162 (Symposium at Davos 1986 - Avalanche Formation, Movement and Effects), 35-48.

Good, W. 1989. Laboratory techniques for the characterization of snow structure. In Hunt, J. and T. D. Guyenne, eds. International Workshop on Physics and Mechanics of Cometary Materials, October 9-11, 1989, Münster, Westfalia, Germany. Proceedings. Noordwijk, European Space Agency, 147-151. (ESA SP-302.)

Hobbs, P.V. 1974. Ice physics. Oxford, Clarendon Press.

Mollard, F. R. and M. C. Flemings. 1967. Growth of composites from the melt (Part 2). Trans. Metall. Soc. AIME, 239, 1534-1546.

Mullins, W.W. and R. F. Sekerka. 1963. Morphological stability of a particle growing by diffusion or heat flow. 7. Appl. Phys., 34, 323-329.

Raymond, C. F. and K. Tusima. 1979. Grain coarsening of water-saturated snow. F. Glaciol., 22 (86), 83-105.

Serra, J. 1982. Image analysis and mathematical morphology. Vol. 1. London, Academic Press.

Sève, R. 1996. Physique de la couleur. Paris, Masson.

Wilcox, W.W. and H. S. Kuo. 1973. Gas bubble nucleation during crystallization. 7. Cryst. Growth, 19, 221-228.

MS received 27 September 1996 and accepted in revised form 26 August 1997 